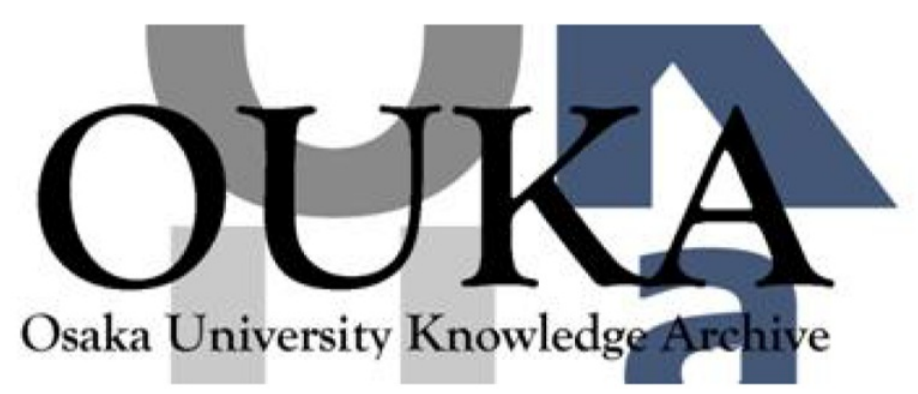

\begin{tabular}{|c|c|}
\hline Title & $\begin{array}{l}\text { Noncontact monitoring of surface-wave } \\
\text { nonlinearity for predicting the remaining life } \\
\text { of fatigued steels }\end{array}$ \\
\hline Author (s) & Ogi, Hirotsugu; Hirao, Masahiko; Aoki, Shinji \\
\hline Citation & Journal of Applied Physics. 90(1) p.438-p.442 \\
\hline Issue Date & 2001-07-19 \\
\hline oaire:version & VoR \\
\hline URL & https://hdl. handle. net/11094/84213 \\
\hline rights & $\begin{array}{l}\text { This article may be downloaded for personal use } \\
\text { only. Any other use requires prior permission } \\
\text { of the author and AIP Publishing. This article } \\
\text { appeared in Journal of Applied Physics, } 90(1) \text {, } \\
438-442 \text { (2001) and may be found at } \\
\text { https://doi.org/10.1063/1.1376668. }\end{array}$ \\
\hline Note & \\
\hline
\end{tabular}

Osaka University Knowledge Archive : OUKA

https://ir. Library. osaka-u. ac. jp/

Osaka University 


\title{
Noncontact monitoring of surface-wave nonlinearity for predicting the remaining life of fatigued steels
}

\author{
Hirotsugu Ogi, ${ }^{\text {a) }}$ Masahiko Hirao, and Shinji Aoki \\ Graduate School of Engineering Science, Osaka University, Toyonaka, Osaka 560-8531, Japan
}

(Received 14 February 2001; accepted for publication 5 April 2001)

\begin{abstract}
A nonlinear acoustic measurement is studied for fatigue damage monitoring. An electromagnetic acoustic transducer (EMAT) magnetostrictively couples to a surface-shear-wave resonance along the circumference of a rod specimen during rotating bending fatigue of carbon steels. Excitation of the EMAT at half of the resonance frequency caused the standing wave to contain only the second-harmonic component, which was received by the same EMAT to determine the second-harmonic amplitude. Thus measured surface-wave nonlinearity always showed two distinct peaks at $60 \%$ and $85 \%$ of the total life. We attribute the earlier peak to crack nucleation and growth, and the later peak to an increase of free dislocations associated with crack extension in the final stage. This noncontact resonance-EMAT measurement can monitor the evolution of the surface-shear-wave nonlinearity throughout the metal's fatigue life and detect the pertinent precursors of the eventual failure. (C) 2001 American Institute of Physics.
\end{abstract}

[DOI: $10.1063 / 1.1376668$ ]

\section{INTRODUCTION}

Acoustic nonlinearity holds the potential of becoming the primary means of characterizing fatigue in materials, because it is capable of probing the processes of crack nucleation and growth ${ }^{1-4}$ and of dislocation movement. ${ }^{5-8}$ Its sensitivity to microstructural attributes during the incubation period is often higher than that of the linear properties (velocity and attenuation). Richardson ${ }^{1}$ analyzed the secondharmonic amplitude generated by the opening and closing effect of an interface subjected to bias pressure by a passing acoustic wave. Optimum pressure exists for secondharmonic generation. Buck et $a l .{ }^{2}$ and Morris et al. ${ }^{3}$ demonstrated that Richardson's model was basically applicable to fatigue cracks in metals; the second-harmonic amplitudes showed maxima with low external compressive stress. Thus, small fatigue cracks act as harmonic generators and the efficiency should exhibit a maximum during crack growth because they are partly closed by compressive residual stress and large cracks are fully open, producing no higher harmonics. With regard to dislocations, Hikata et al. ${ }^{5}$ studied the harmonic generation caused by their anelastic vibration and showed that the second-harmonic amplitude increases with an increase of dislocation density and loop length. We recently revealed that a dislocation structure change occurs in the later stages of rotating bending fatigue, during which many mobile dislocations temporally arise. ${ }^{9,10}$ These theoretical and experimental studies predicted a nonlinearity peak as a result of this event.

We expect then two nonlinear peaks during a fatigue life, one associated with cracks and the other with dislocations. However, such a study has not been reported in the literature. We note two key points. First, surface waves should be used to focus on the specimen surfaces, where

\footnotetext{
${ }^{a)}$ Electronic mail: ogi@me.es.osaka-u.ac.jp
}

damage progresses. Second, a contactless acoustic transduction should be used to avoid background nonlinearity caused by coupling agents and the transducer itself. Of the previous nonlinear acoustic studies for materials characterization, few satisfied these requisites and none detected fatigue damage. A notable exception was reported by Hurley, ${ }^{11}$ who used a comb transducer for excitation and an interferometric laser detector to accurately measure Rayleigh wave harmonics in aluminum, a quasicontactless technique.

In the present study, we apply an electromagnetic acoustic transducer (EMAT) to monitor the surface-shear-wave nonlinearity throughout the metal's fatigue life. The use of an EMAT makes contactless transduction possible. But, EMATs lack a large transduction efficiency, which the nonlinear measurement needs. To overcome this limitation, we chose to use acoustic resonance so as to excite and enhance the standing wave of the second-harmonic component around a rod specimen. Coherent superposition produces a highly magnified amplitude of the second harmonics. Thus measured surface-wave nonlinearity detected the two peaks in the fatigue life.

\section{MATERIALS}

We used commercial steel rods containing 0.25 or 0.35 mass percent carbon. They were heated at $880^{\circ} \mathrm{C}$ for $1 \mathrm{~h}$ and cooled in air. The specimens were $600 \mathrm{~mm}$ long. Their diameter was $14 \mathrm{~mm}$ at the center; it smoothly increased from 14 to $20 \mathrm{~mm}$ with a large curvature to bring about damage and failure at the minimum diameter. We prepared the measuring surface by electropolishing.

\section{RESONANCE-EMAT METHOD FOR NONLINEAR ACOUSTICS}

\section{A. Spectroscopy measurement}

The EMAT consists of a solenoid coil to introduce the bias magnetic field $\mathbf{H}_{0}$ in the specimen's axial direction and 


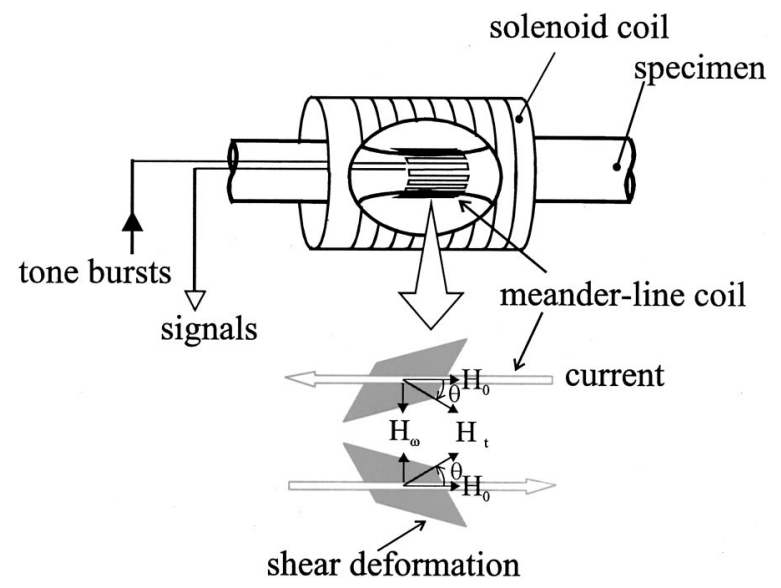

FIG. 1. Configuration and mechanism of the magnetostrictive EMAT for generating and receiving the axial shear wave.

a meander-line coil, having equal meandering periods of 0.9 $\mathrm{mm}$, to induce the dynamic field $\mathbf{H}_{\omega}$ in the circumference direction (see Fig. 1). When sinusoidal current is applied to the meander-line coil, the total field $\mathbf{H}_{t}$ oscillates about the axial direction at the same frequency as the driving current and produces shearing vibration through the magnetostriction effect to excite the surface-shear wave propagating along the circumference with axial polarization. ${ }^{12}$ This is called the axial shear wave. ${ }^{13}$ After excitation, the same meander-line coil receives the axial shear wave through the reversed magnetostrictive effect.

Driving the meander-line coil with long tone bursts causes interference among the axial shear waves traveling around the cylindrical surface, and a frequency scan detects resonance peaks at unequal frequency intervals, at which all the waves overlap coherently to produce large amplitudes. We used the first resonance mode around $f_{r}=3.9 \mathrm{MHz}$, whose amplitude distribution has the maximum at the surface and a steep gradient with the radius; the penetration depth is estimated to be $0.5 \mathrm{~mm} .{ }^{10}$ We defined the maximum amplitude of the first resonance peak as the fundamental amplitude, $A_{1}$ (Fig. 2). We then excited the axial shear wave by driving the EMAT at half of the resonance frequency $\left(f_{r} / 2\right)$, keeping the input power unchanged. In this case, the driving frequency does not satisfy the resonance condition and the

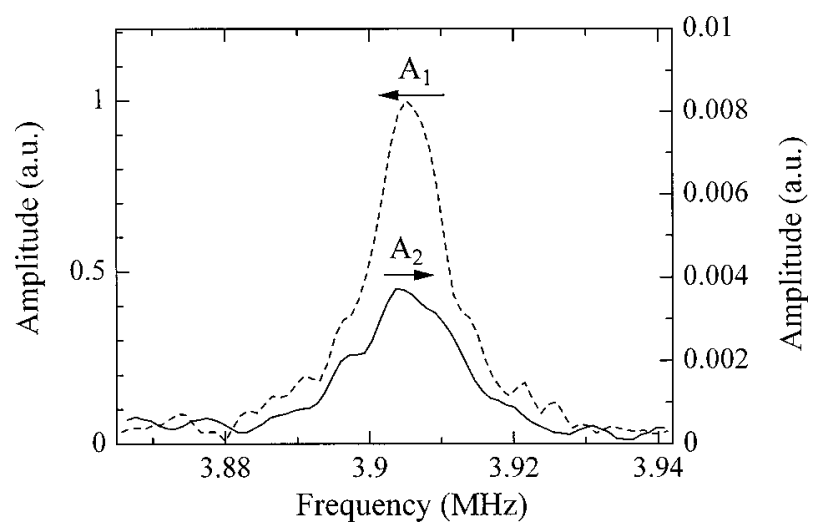

FIG. 2. Resonance spectra for the fundamental and the second-harmonic components.

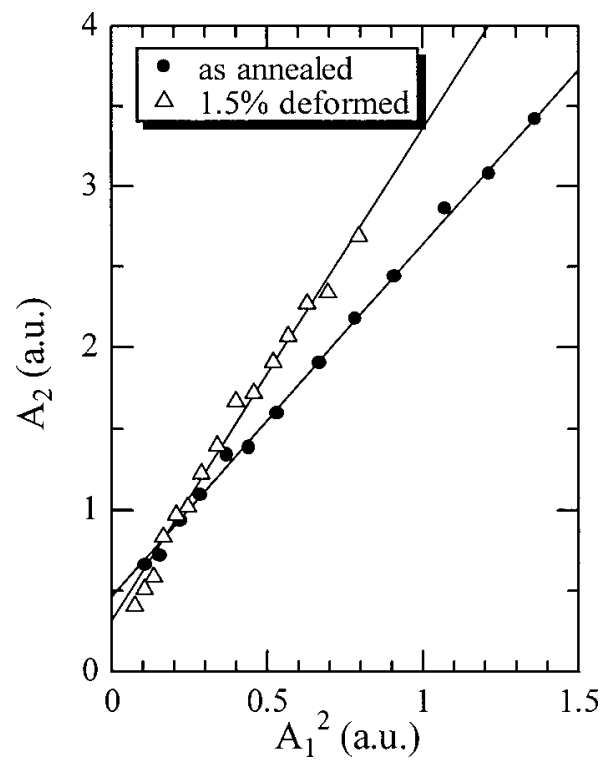

FIG. 3. Verification of the second power law before and after plastic elongation. The second-harmonic amplitude is proportional to the square of the fundamental amplitude.

fundamental component does not produce a detectable signal. However, the second-harmonic component having double frequency $\left(f_{r}\right)$ satisfies the resonance condition and the resonance spectrum of the received signal contains a peak at the original resonance frequency as shown in Fig. 2. We defined this peak height as the second-harmonic amplitude, $A_{2}$, to calculate the nonlinearity $A_{2} / A_{1}$. The magnitude of $A_{2} / A_{1}$ varied on the order of $10^{-3}$. These measurements were made possible by the system for nonlinear acoustic phenomena (SNAP) manufactured by RITEC Inc., which provided the meander-line coil with the burst signal and detected the resonance peaks.

\section{B. Second power law}

In metals without flaws, higher harmonics arise from nonlinear elasticity due to lattice anharmonicity and from anelasticity due to dislocation movement. These two effects are inseparable in actual nonlinear measurements. Both generate higher harmonics, among which the second harmonic usually dominates. The amplitude is then given by the square of the fundamental wave amplitude, ${ }^{5}$ that is, second power law.

The present definition of nonlinearity $A_{2} / A_{1}$ is based on second power law and the proportionality between $A_{1}\left(f_{r}\right)$ and the true fundamental amplitude, say, $A_{1}^{\prime}\left(f_{r} / 2\right)$. The second-harmonic amplitude $A_{2}$ is of course proportional to the square of the true fundamental amplitude $A_{1}^{\prime}$, that is, $A_{2} \propto A_{1}^{\prime 2}$. This component lasts only a very short time after excitation and vanishes through mutual interference in the off-resonance condition. Within this short time, it generates the second harmonics following second power law. But, we cannot measure $A_{1}^{\prime}$. Instead, we measured $A_{1}$ and $A_{2}$, both at $f_{r}$, by changing the driving voltage. Figure 3 shows the result for 0.25 mass $\% \mathrm{C}$ steel before and after applying $1.5 \%$ tensile plastic deformation. It demonstrates the linear rela- 


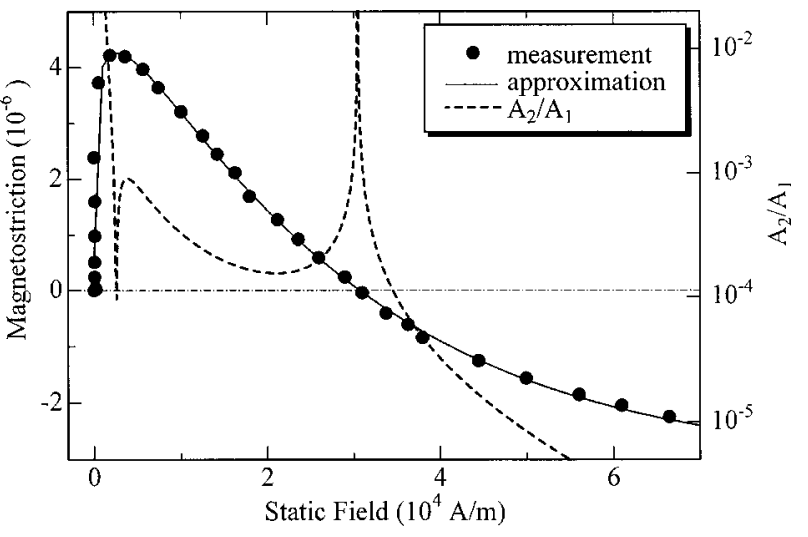

FIG. 4. Measured magnetostriction (closed circle), the fitted function (solid line), and the calculated nonlinearity in transmission (broken line). The 0.25 mass \% $\mathrm{C}$ steel was used after demagnetization.

tionship between $A_{2}$ and $A_{1}^{2}$, proving that $A_{1} \propto A_{1}^{\prime}$. (The larger slope after deformation is caused by dislocation multiplication. The slope is proportional to the dislocation density $\Lambda$ times the fourth power of the loop length $L\left(A_{2} / A_{1}^{2}\right.$ $\left.\propto \Lambda L^{4}\right) .^{5}$ ) Normalization of $A_{2}$ in terms of $A_{1}$ removes the influences of liftoff, frequency dependence of the transduction efficiency, and other anomalies.

\section{Background nonlinearity by the magnetostrictive effect}

Magnetostrictively coupling EMAT entails background nonlinearity caused by the metal's magnetostrictive response to the applied magnetic field. We have to estimate this effect. A preceding study ${ }^{14}$ derived a relationship between the bulkshear-wave amplitude and the metal's magnetostrictive characteristics by assuming isovolume magnetostriction and reversible magnetization rotation. A similar approach is possible in the present situation by assuming a flat surface. The resultant surface-shear-wave amplitude, $U_{\mathrm{SH}}$, is related to the magnetostriction $\epsilon_{M}$ and its field derivative $\left(d \epsilon_{M} / d H\right)$ as

$$
\begin{aligned}
U_{\mathrm{SH}} \propto & \frac{3 \mu}{H_{0}}\left\{\epsilon_{M} \cos 2 \theta \cos ^{2} \theta\right. \\
& \left.+3 H_{0}\left(\frac{d \epsilon_{M}}{d H}\right) \sin ^{2} \theta \cos \theta\right\} e^{j \omega t} .
\end{aligned}
$$

Here, $\mu$ denotes the shear modulus, $\theta=\tan ^{-1}\left(H_{\omega} / H_{0}\right)$ (see Fig. 1), and $H_{\omega}=h_{\omega} \exp (j \omega t)$ with the dynamic-field amplitude $h_{\omega}$. When the static field $H_{0}$ is much larger than the dynamic field $H_{\omega}$, i.e., $|\theta(t)| \ll 1$, Eq. (1) is approximated as

$$
U_{\mathrm{SH}} \propto \frac{3 \mu}{H_{0}}\left\{\epsilon_{M}+3\left(\frac{d \epsilon_{M}}{d H}\right) \frac{h_{\omega}^{2}}{H_{0}} e^{j \omega t}\right\} e^{j \omega t} .
$$

The amplitude ratio $A_{2} / A_{1}$ is then given by

$$
\frac{A_{2}}{A_{1}}=\left|\frac{3}{\epsilon_{M}}\left(\frac{d \epsilon_{M}}{d H}\right)_{H=H_{0}}\right| \frac{h_{\omega}^{2}}{H_{0}} .
$$

Figure 4 shows the magnetostriction measured by chang- ing the applied field with 0.25 mass \% C steel. We obtained the fitting function to the measurements

$$
\epsilon_{M}(H)=3 H^{0.15} \exp (-0.457 H+1.14)-2.75 \text {, }
$$

with $\epsilon_{M}$ in microstrain $\left(10^{-6}\right)$ and $H$ in $10^{4} \mathrm{~A} / \mathrm{m}$.

Thompson ${ }^{15}$ derived the magnitude of the tangential dynamic-field amplitude $h_{\omega}$ induced by driving a meanderline coil, which, in first approximation, reduces to

$$
h_{\omega}=\frac{2 I}{D} \frac{\sin (\pi a / D)}{\pi a / D} \exp \left(-\frac{2 \pi G}{D}\right),
$$

where $I$ denotes the current, $D$ the meander-line period, $a$ the width of the coil lines, and $G$ the air gap between the coil and specimen surface. In this study, $D=0.9 \mathrm{~mm}$ and $a$ $=0.01 \mathrm{~mm}$. Substitution of Eqs. (4) and (5) into Eq. (3) results in the normalized second-harmonic amplitude $A_{2} / A_{1}$ in Fig. 4 for the typical values of $I=0.5 \mathrm{~A}$ and $G=0.3 \mathrm{~mm}$. We see the infinite transduction nonlinearity at zero static field and also at $H_{0} \sim 3.1 \times 10^{4} \mathrm{~A} / \mathrm{m}$, both of which result from zero magnetostriction. An ideal static field should provide large transduction efficiency and minimum nonlinearity. We fixed the static field at $H_{0}=1.2 \times 10^{4} \mathrm{~A} / \mathrm{m}$ throughout this study so that the background nonlinearity remains one order of magnitude smaller than the fatigue-induced nonlinearity, keeping sufficient efficiency (the efficiency is nearly proportional to $\epsilon_{M}$ ). It should be noted that the background nonlinearity continues to be unchanged during the fatigue test so long as a constant static field is applied.

\section{ROTATING BENDING FATIGUE}

\section{A. Measurements}

The measurement setup of the rotating bending fatigue test was the same as that developed in our previous study. ${ }^{10}$ The meander-line coil surrounded the specimen's center part. We rotated the specimen at $240 \mathrm{rpm}(4 \mathrm{~Hz})$. A four-point bending configuration gave a maximum bending stress of $280 \mathrm{MPa}\left(=0.84 \sigma_{y}, \sigma_{y}\right.$ : yield strength) for 0.25 mass $\% \mathrm{C}$ steel and 357 and $382 \mathrm{MPa}$ for 0.35 mass \% C steel (0.79 and $0.85 \sigma_{y}$, respectively). The cycle to failure, $N_{F}$, was of the order of $10^{4}$. We measured the nonlinearity, attenuation, and phase velocity of the surface-shear wave by interrupting the cyclic loading and releasing the bending stress. Details of the velocity and attenuation measurements appear in Ref. 16. Along with the acoustic measurements, we observed surfacecrack nucleation and growth by replication. ${ }^{10} \mathrm{We}$ then restarted the cyclic loading. This procedure was repeated until failure.

\section{B. Results}

Figures 5(a) and 5(b) show typical evolutions of the phase velocity $\nu$, the attenuation coefficient $\alpha$, and the normalized nonlinearity $A_{2} / A_{1}$ for the first axial-shear-wave resonance. Individual measurements of $A_{1}$ and $A_{2}$ are plotted in Fig. 5(c). The number of cycles, $N$, is normalized by $N_{F}$. Measurements of the linear characteristics (velocity and attenuation) are consistent with those observed in our previous study for 0.45 mass \% C steel and a 5052 aluminum alloy with the same specimen dimensions: ${ }^{10}$ the attenuation coef- 


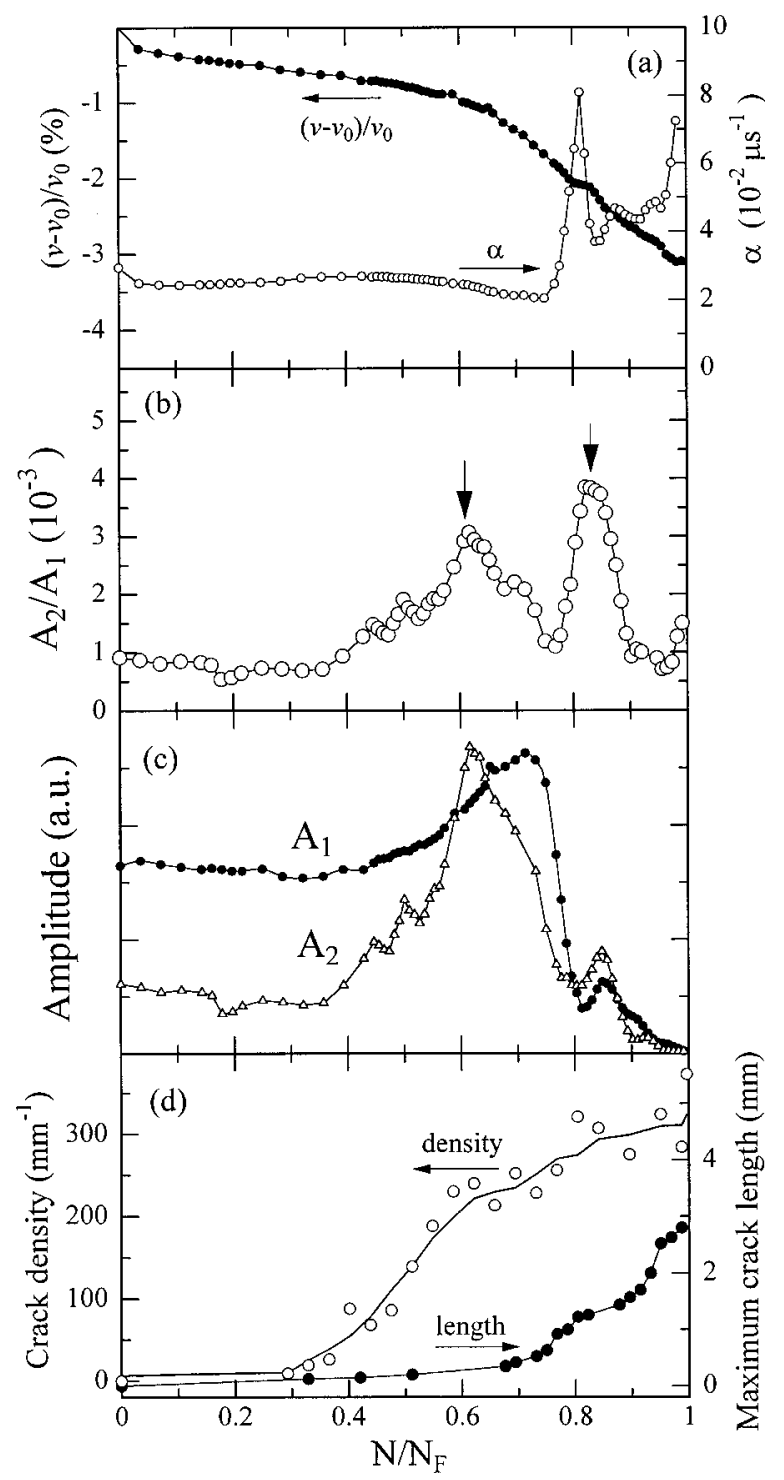

FIG. 5. Typical evolutions of (a) velocity and attenuation, (b) the nonlinearity, (c) the fundamental and second-harmonic amplitudes, and (d) surface cracks for 0.25 mass \% C steel $\left(N_{F}=56000\right)$. Arrows indicate the first and the second nonlinearity peaks.

ficient always shows a sharp peak at $85 \%$ of the total life, which is accompanied by the deceleration of a velocity decrease. Important observations here are (i) the nonlinearity shows two peaks during the fatigue life; the first one appears around $60 \%$ and the second one around $85 \%$ of $N_{F}$, almost the same time as the attenuation peak; (ii) this is independent of the carbon content and the bending stress (see Fig. 6).

Figure 5(d) shows the result of the surface crack observation. The crack density was calculated from the total crack length divided by the viewing area. Cracks were observable with an optical microscope as early as at about $30 \%$ of $N_{F}$, the average length being about $50 \mu \mathrm{m}$ at this stage. The crack density increased linearly with $N$, while the maximum length remained unchanged until about $70 \%$, which was followed by a rapid increase toward failure. This observation indicates that cyclic loading between $N / N_{F}=0.3$ and 0.7 was expended mainly by crack nucleation, not by crack growth, and produced many small cracks of uniform size. This ap-

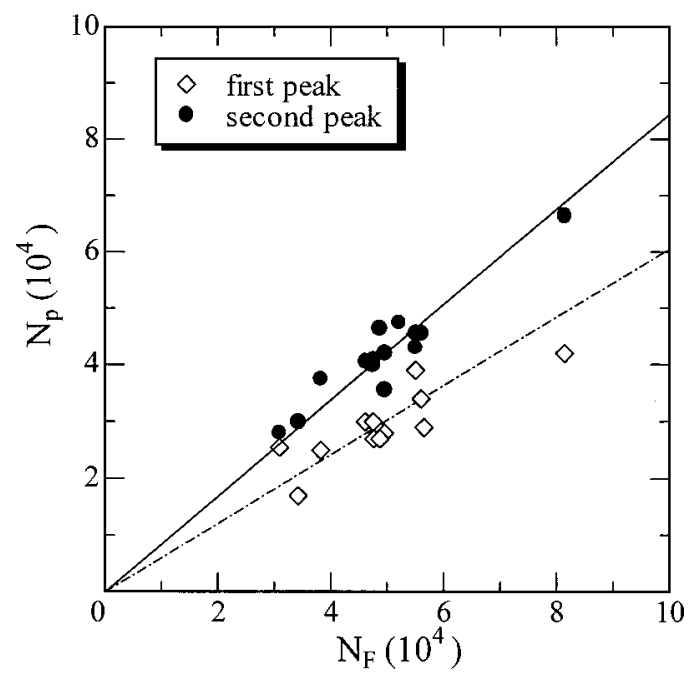

FIG. 6. Relationship between the failure cycle number $N_{F}$ and the cycle numbers at the first and the second nonlinearity peaks $\left(N_{P}\right)$ for 0.25 and 0.35 mass \% C steels.

pears reasonable considering the metal's work hardening and the bending stress gradient. After $N / N_{F}=0.7$, these small cracks coalesce with each other to form longer cracks, resulting in growth inward in the final stage and then fracture.

\section{DISCUSSIONS}

Detailed investigation of the attenuation peak ${ }^{10}$ and observations of the dislocation structure by transmission electron micrography ${ }^{17}$ revealed that the attenuation peak responds to a temporal increase of free dislocations. According to the string model of vibrating dislocations, ${ }^{18} \alpha\left(\propto \Lambda L^{4}\right)$ increases and $v\left(\infty-\Lambda L^{2}\right)$ decreases with an increase of free dislocations that vibrate with the acoustic wave. The crack coalescence at the later stage produces high stress zones ahead of the crack tips and gives rise to rearrangement of the dislocation cell structure, the release of pinned dislocations from obstacles, and dislocation multiplication. This involves the production of free dislocations, which raises $\alpha$ several times from the initial measurement. The attenuation coefficient, however, decreases soon because of the dislocation tangling and piling up again. This process takes place simultaneously at many sites within the thin surface layer of the specimen. The velocity change can be interpreted in the same way, although it is less sensitive to the microstructure.

We interpret the second (later) nonlinear peak as caused by the above crack-dislocation interaction because it synchronized with the attenuation peak. Anelastic behavior of the free dislocations is the common source of attenuation and nonlinearity. A supplemental experiment of lowering the free dislocations confirms this viewpoint (Fig. 7). We stopped the fatigue test at the second nonlinearity peak, exposed the specimen to $300^{\circ} \mathrm{C}$ for $1 \mathrm{~h}$, and observed a drop in nonlinearity to that before the peak. The attenuation coefficient and the velocity also returned to the previous values as a result of this heat treatment.

We attribute the first nonlinearity peak to crack nucleation and growth. Small fatigue cracks are partly closed along the tips due to compressive residual stress, which 


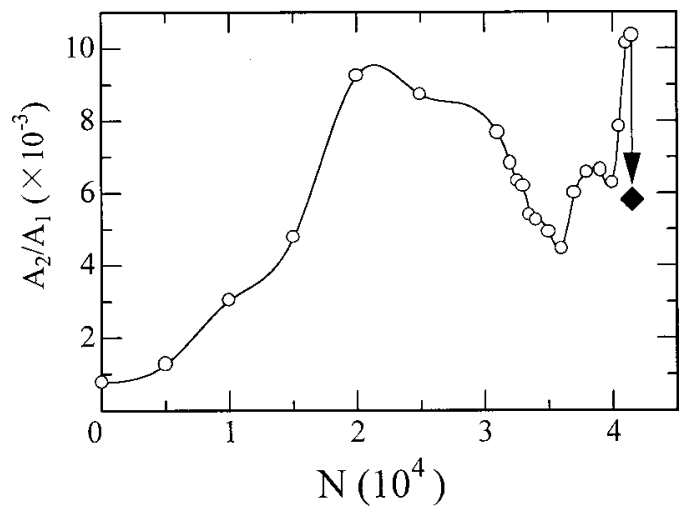

FIG. 7. Recovery of nonlinearity from the second peak by annealing at $300^{\circ} \mathrm{C}$ for $1 \mathrm{~h}$.

arises from local plastic deformation (plasticity-induced closure). ${ }^{19}$ The residual stress is large near the tip, which tightly closes the crack faces. It diminishes as it approaches the crack mouth and there is a thin band where the crack faces are in contact at very small pressure. ${ }^{3}$ These thin bands open and close when the acoustic wave impinges on them, and they distort the waveform, making a strong nonlinearity source. In the present case, they are at many sites, since the crack shape is shallow and semiellipsoidal with relatively uniform size. The nonlinearity should then increase as the number of small cracks increases. In Fig. 5, the crack density correlates with the nonlinearity prior to the first peak, where the crack length remains unchanged and the number of cracks monotonically increases. In the course of fatigue, the small cracks coalesce with each other and start to grow inward. This process reduces the area of weakly touching crack faces because of the asperity contact across the shallower faces, resulting in a drop of nonlinearity. The crack length starts to increase after this nonlinearity peak in Fig. 5(d), being compatible with the above interpretation. Thus, the first nonlinearity peak indicates the beginning of crack coalescence.

We finally want to remark on the effect of attenuation in the nonlinearity of $A_{2} / A_{1}$. Roughly speaking, $\alpha$ and $A_{1}$ vary in reversed ways in Fig. 5, implying that attenuation governs $A_{1}$. But, $A_{2}$ is independent of the attenuation variation and $A_{2}$ dominates in the first nonlinearity peak. The amplitude $A_{2}$ originates from $A_{1}^{\prime}$, which is considered to be constant throughout the fatigue tests since we used equal input power to the EMAT. Indeed, no link is found between $A_{1}$ and $A_{2}$ until the attenuation peak. After that, they change in a similar way, indicating the common influence of dislocations. The normalization by $A_{1}$ is still useful because the electromagnetic properties of metal, such as conductivity, may change during fatigue and affect the EMAT's transduction efficiency.
The most important observation in this study is that the nonlinearity peaks appear at fixed fractions to $N_{F}$ in Fig. 6 . The same is true for the indicative decline in the phase velocity $v$ and the sharp peak of the attenuation coefficient $\alpha$. These measurements detect the pertinent microstructural changes inside the metal, which closely correlate with the degradation process and lead to the final fracture.

\section{CONCLUSION}

We summarize our conclusions as the following.

(1) Combination of the magnetostrictive EMAT and the resonance method enables us to detect the second-harmonic amplitude of the surface-shear wave without contact.

(2) The measurement of acoustic nonlinearity we have used obeys the second power law and shows the expected sensitivity to cracks and dislocations.

(3) The nonlinearity shows two distinct peaks during rotating bending fatigue of carbon steels at $60 \%$ and $85 \%$ of the total life. These percentage lives are unchanged in the stress region of $0.79-0.85$ of the yield strength. The resonance-EMAT method holds promise then for developing into a means by which we predict the remaining life of a fatigued steel.

(4) The first nonlinearity peak responds to crack nucleation and then coalescences, which is supported by the surface-crack observation.

(5) The second nonlinearity peak is synchronized with the attenuation peak and is induced by the temporal increase of free dislocations.

${ }^{1}$ J. M. Richardson, Int. J. Eng. Sci. 17, 73 (1979).

${ }^{2}$ O. Buck, W. L. Morris, and J. M. Richardson, Appl. Phys. Lett. 33, 371 (1978).

${ }^{3}$ W. L. Morris, O. Buck, and R. V. Inman, J. Appl. Phys. 50, 6737 (1979).

${ }^{4}$ P. B. Nagy, Ultrasonics 36, 375 (1998).

${ }^{5}$ A. Hikata, B. Chick, and C. Elbaum, J. Appl. Phys. 36, 229 (1965).

${ }^{6}$ A. Hikata and C. Elbaum, Phys. Rev. 144, 469 (1966).

${ }^{7}$ A. Hikata, F. A. Sewell, Jr., and C. Elbaum, Phys. Rev. 151, 442 (1966).

${ }^{8}$ J. H. Cantrell and W. T. Yost, Philos. Mag. A 69, 315 (1994).

${ }^{9}$ H. Ogi, M. Hirao, and K. Minoura, J. Appl. Phys. 81, 3677 (1997).

${ }^{10}$ H. Ogi, T. Hamaguchi, and M. Hirao, Metall. Mater. Trans. A 31A, 1121 (2000).

${ }^{11}$ D. C. Hurley, J. Acoust. Soc. Am. 106, 1782 (1999).

${ }^{12}$ H. Ogi, M. Hirao, and K. Minoura, Review of Progress in QNDE, edited by D. O. Thompson and D. E. Chimenti (Plenum, New York, 1996), Vol. 15, pp. 1939-1944.

${ }^{13}$ W. Johnson, B. A. Auld, and G. A. Alers, Review of Progress in QNDE, edited by D. O. Thompson and D. E. Chimenti (Plenum, New York, 1994), Vol. 13, pp. 1603-1609.

${ }^{14}$ H. Ogi, J. Appl. Phys. 82, 3940 (1997)

${ }^{15}$ R. B. Thompson, Physical Acoustics, edited by R. N. Thurston and A. D. Pierce (Academic, New York, 1990), Vol. 19, pp. 157-200.

${ }^{16}$ H. Ogi, M. Hirao, and T. Honda, J. Acoust. Soc. Am. 98, 458 (1995).

${ }^{17}$ H. Ogi, Y. Minami, and M. Hirao (unpublished).

${ }^{18}$ A. Granato and K. Lücke, J. Appl. Phys. 27, 583 (1956).

${ }^{19}$ Mechanics of Fatigue Crack Closure, edited by J. C. Newman, Jr. and W. Elber ASTM STP 982 (American Society for Testing Materials, Philadelphia, PA, 1988) 